\title{
Evaluation of three-dimensional biofilms on antibacterial bonding agents containing novel quaternary ammonium methacrylates
}

\begin{abstract}
Han Zhou ${ }^{1,2}$, Michael D Weir ${ }^{1}$, Joseph M Antonucci ${ }^{3}$, Gary E Schumacher ${ }^{4}$, Xue-Dong Zhou ${ }^{2}$ and Hockin H K Xu ${ }^{1,5,6}$
Antibacterial adhesives are promising to inhibit biofilms and secondary caries. The objectives of this study were to synthesize and incorporate quaternary ammonium methacrylates into adhesives, and investigate the alkyl chain length effects on three-dimensional biofilms adherent on adhesives for the first time. Six quaternary ammonium methacrylates with chain lengths of 3, 6, 9, 12, 16 and 18 were synthesized and incorporated into Scotchbond Multi-Purpose. Streptococcus mutans bacteria were cultured on resin to form biofilms. Confocal laser scanning microscopy was used to measure biofilm thickness, live/dead volumes and live-bacteria percentage vs. distance from resin surface. Biofilm thickness was the greatest for Scotchbond control; it decreased with increasing chain length, reaching a minimum at chain length 16 . Live-biofilm volume had a similar trend. Dead-biofilm volume increased with increasing chain length. The adhesive with chain length 9 had 37\% live bacteria near resin surface, but close to $100 \%$ live bacteria in the biofilm top section. For chain length 16 , there were nearly $0 \%$ live bacteria throughout the three-dimensional biofilm. In conclusion, strong antibacterial activity was achieved by adding quaternary ammonium into adhesive, with biofilm thickness and live-biofilm volume decreasing as chain length was increased from 3 to 16 . Antibacterial adhesives typically only inhibited bacteria close to its surface; however, adhesive with chain length 16 had mostly dead bacteria in the entire three-dimensional biofilm. Antibacterial adhesive with chain length 16 is promising to inhibit biofilms at the margins and combat secondary caries.
\end{abstract}

International Journal of Oral Science (2014) 6, 77-86; doi:10.1038/ijos.2014.18; published 11 April 2014

Keywords: alkyl chain length; antibacterial bonding agent; dental caries; quaternary ammonium methacrylate; Streptococcus mutans; three-dimensional biofilm

\section{INTRODUCTION}

Dental resin composites are the principal materials for tooth cavity restorations due to their excellent esthetics and direct-filling capabilities. ${ }^{1-4}$ Composite restorations are bonded to tooth structures via bonding agents. ${ }^{5-7}$ Studies have shown that secondary caries is one of the primary reasons for restoration failure. ${ }^{3,5,8-9}$ and replacing the failed restorations accounts for $50 \%-70 \%$ of all restorations performed. ${ }^{10-11}$ The cause of caries is biofilm acid production; ${ }^{12}$ therefore, efforts were made to develop antibacterial resins. ${ }^{13-14}$ Quaternary ammonium methacrylates (QAMs) were synthesized and copolymerized in dental resins to obtain antibacterial functions. ${ }^{15-23}$ Because residual bacteria often exist in the prepared tooth cavity and microleakage could allow new bacteria to invade the tooth-restoration interface, it would be especially useful for the bonding agent to possess antibacterial functions. ${ }^{13-16,24-25}$

Quaternary ammonium salts (QAS) can cause bacteria lysis by binding to bacterial membranes. ${ }^{17,26}$ When the negatively charged bacterial cell contacts the positively charged sites of the quaternary ammonium, the electric balance of the cell membrane could be disturbed, and the bacterium could be damaged or killed. ${ }^{17,26}$ The long polymeric chains with positive charges appeared to contribute to the efficacy of bactericide. ${ }^{27}$ Increasing the alkyl chain length (CL) increased the hydrophobicity, which could enhance the propensity to penetrate the hydrophobic bacterial membrane. ${ }^{28}$ Therefore, cationic polymers with longer CL could be more effective in penetrating bacterial cells to disrupt membranes. ${ }^{28-29}$ However, to date, there has been no report on the effect of CL of QAMs in dental bonding agents, except a recent study. ${ }^{30}$ That study synthesized a series of new QAMs with CL from 3 to 18 , incorporated them into dental adhesive and achieved strong antibacterial efficacy, without compromising the dentin bond strength. ${ }^{30}$ However, the three-dimensional (3D) biofilm structure and live/dead bacteria viability distribution along biofilm thickness $v$ s. CL were not investigated.

Previous studies on dental biofilms were performed using light, scanning and transmission electron microscopy which provided

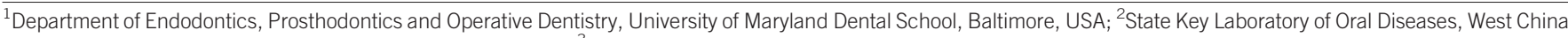

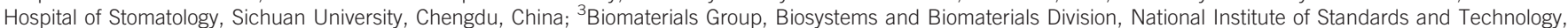

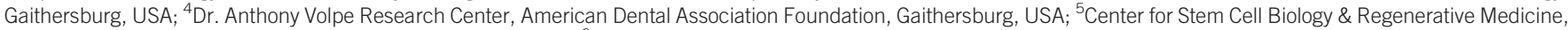
University of Maryland School of Medicine, Baltimore, USA and ${ }^{6}$ Department of Mechanical Engineering, University of Maryland, Baltimore County, USA

Correspondence: Professor HHK Xu, Department of Endodontics, Prosthodontics and Operative Dentistry, University of Maryland Dental School, Baltimore MD 21201, USA

E-mail: hxu@umaryland.edu

Professor XD Zhou, State Key Laboratory of Oral Diseases, West China Hospital of Stomatology, Sichuan University, Chengdu 610041, China

E-mail: zhouxd@scu.edu.cn

Accepted 26 January 2014 
two-dimensional (2D) view of the top surface of the biofilm or the structure of a single cell. ${ }^{31-32}$ Besides these established techniques, the application of confocal laser scanning microscopy (CLSM) was also introduced in dental research for assessment of oral biofilms. ${ }^{33-37}$ CLSM allows horizontal and vertical optical sectioning of the 3D biofilm. The $3 \mathrm{D}$ biofilm structure can be reconstructed from $2 \mathrm{D}$ images of thin sections throughout the biofilm. Image-processing techniques are used for quantitative analysis of biofilms to obtain a detailed visualization of thick biofilm samples, ${ }^{38}$ which cannot be obtained via conventional phase contrast or fluorescence microscopy. Previous studies used CLSM techniques to analyze the 3D viability distribution in dental biofilms. ${ }^{36-37,39-40}$ However, there has been no report on the effect of $\mathrm{CL}$ on $3 \mathrm{D}$ viability distribution of biofilms adherent on dental bonding agents. It would be interesting to know: (i) how CL would affect biofilm thickness and live/dead biofilm volumes on adhesive resins; and (ii) if there would be more dead bacteria near the antibacterial bonding agent surface, and less dead bacteria in the biofilm further away from bonding agent surface.

Therefore, the objectives of this study were to incorporate QAMs into dental bonding agent, and investigate the effects of CL on the 3D biofilm structure, live biofilm volume and viability distribution along biofilm thickness adherent on dental bonding agents for the first time. It was hypothesized that: (i) the viability of 3D biofilms growing on dental adhesive containing QAM will decrease with increasing CL; (ii) CL of QAM in adhesive resin will have a significant effect on biofilm thickness and live and dead biofilm volumes; (iii) there will be less live bacteria in the biofilm near antibacterial bonding agent surface, and the percentage of live bacteria in the biofilm will increase with increasing distance away from bonding agent surface.

\section{MATERIALS AND METHODS}

\section{Synthesis of antibacterial QAMs with different chain length CL}

New QAMs were synthesized using a modified Menschutkin reaction via the addition reaction of a tertiary amine with an organohalide. ${ }^{20-23}$ A benefit of this reaction is that the reaction products are generated at virtually quantitative amounts and require minimal purification. ${ }^{20}$ The 2-(dimethylamino) ethyl methacrylate (DMAEMA; Sigma Aldrich, St Louis, MO, USA) was the methacrylate-containing tertiary amine. For example, to synthesize dimethylaminododecyl methacrylate (DMADDM) with $\mathrm{CL}=12,10 \mathrm{mmol}$ of DMAEMA, $10 \mathrm{mmol}$ of 1 bromododecane (TCI America, Portland, OR, USA) and $3 \mathrm{~g}$ of ethanol were added to a vial, which was capped and stirred at $70{ }^{\circ} \mathrm{C}$ for $24 \mathrm{~h}^{41}$ After the reaction was completed, the ethanol was removed via evaporation. This yielded DMADDM as a clear liquid, which was verified via Fourier transform infrared spectroscopy in a recent study. ${ }^{41}$ Using this method, six QAMs with CL of 3, 6, 9, 12, 16 and 18 were synthesized, ${ }^{30}$ namely:

(i) DMAEMA was reacted with 1-bromopropane to form dimethylaminopropyl methacrylate (DMAPM, CL=3).

(ii) DMAEMA was reacted with 1-bromohexane to form dimethylaminohexyl methacrylate (DMAHM, CL=6).

(iii) DMAEMA was reacted with 1-bromononane to form dimethylaminononyl methacrylate (DMANM, CL=9).

(iv) DMAEMA was reacted with 1-bromododecane to form DMADDM $(\mathrm{CL}=12)$.

(v) DMAEMA was reacted with 1-bromohexadecane to form dimethylaminohexadecyl methacrylate (DMAHDM, CL = 16).

(vi) DMAEMA was reacted with 1-bromooctadecane to form dimethylaminooctadecyl methacrylate (DMAODM, CL=18).

\section{Processing of antibacterial bonding agents}

To formulate antibacterial bonding agents, Scotchbond multi-purpose bonding agent (SBMP; 3M, St Paul, MN, USA) was used as the parent system. According to the manufacturer, SBMP adhesive contained $60 \%-70 \%$ of bisphenol A diglycidyl methacrylate and $30 \%-40 \%$ of 2 -hydroxyethyl methacrylate, tertiary amines and photo-initiator. SBMP primer contained $35 \%-45 \%$ of 2 -hydroxyethyl methacrylate, $10 \%-20 \%$ of a copolymer of acrylic and itaconic acids and $40 \%-50 \%$ water. Each QAM was mixed SBMP primer at a QAM/ (SBMP primer+QAM) mass fraction of $10 \%$, following previous studies. $^{22-23,30}$ SBMP adhesive was also incorporated with $10 \%$ QAM. $^{22-23,30}$ This yielded six antibacterial bonding agents, corresponding to the six QAMs. They are designated, respectively, as:
(i) $\mathrm{SBMP}+\mathrm{DMAPM}(\mathrm{CL} 3)$;
(ii) $\mathrm{SBMP}+\mathrm{DMAHM}$ (CL6);
(iii) SBMP+DMANM (CL9);
(iv) SBMP+DMADDM (CL12);
(v) SBMP+DMAHDM (CL16);
(vi) SBMP+DMAODM (CL18).

\section{Fabrication of resin specimens for biofilm culture}

Resin disks for biofilm experiments were fabricated using the cover of a sterile 96 -well plate as molds. ${ }^{16}$ Following previous studies, ${ }^{16,30}$ $10 \mu \mathrm{L}$ primer was brushed onto the bottom of each dent of approximately $8 \mathrm{~mm}$ in diameter. The primer was dried with a stream of air and then $20 \mu \mathrm{L}$ of adhesive was applied. A Mylar strip was used to covered on the adhesive which was then light-cured for $20 \mathrm{~s}$ (Optilux VCL 401; Demetron Kerr, Danbury, CT, USA). This yielded a cured resin disk of approximately $8 \mathrm{~mm}$ in diameter and $0.5 \mathrm{~mm}$ in thickness. $^{30}$ The disks were removed from the cover of the 96 -well plate, immersed in $200 \mathrm{~mL}$ of distilled water and stirred via a magnetic stirrer (Bellco Glass, Vineland, NJ, USA) at a speed of $100 \mathrm{r} \cdot \mathrm{min}^{-1}$ for $1 \mathrm{~h}$ to remove any uncured monomers, following previous studies. ${ }^{15}$ The disks were then dried, sterilized in an ethylene oxide sterilizer (Anprolene AN 74i; Andersen, Haw River, NC, USA) and then de-gassed for 7 days following manufacturer's instructions.

The use of Streptococcus mutans (S. mutans) bacteria (ATCC700610; American Type, Manassas, VA, USA) was approved by the Institutional Review Board of the University of Maryland. ${ }^{21}$ A $15 \mu \mathrm{L}$ of S. mutans stock bacteria was added to $15 \mathrm{~mL}$ of brain heart infusion broth (Becton, Sparks, MD, USA) and incubated at $37{ }^{\circ} \mathrm{C}$ with $5 \% \mathrm{CO}_{2}$ for $16 \mathrm{~h} ; 150 \mu \mathrm{L}$ of this $S$. mutans suspension was then diluted by 10 fold in a growth medium which consisted of brain heart infusion supplemented with $0.2 \%$ sucrose to form $S$. mutans inoculation medium of $1.5 \mathrm{~mL}$.

\section{CLSM analysis of biofilms}

Seven bonding agent groups were tested: the six bonding agents containing QAM with different CLs and the unmodified SBMP as control. Six disks $(n=6)$ were used for each bonding agent, requiring a total of 42 disks. Each disk was placed in a well of a 24 -well plate and inoculated with $1.5 \mathrm{~mL}$ of the $S$. mutans inoculation medium. The samples were incubated at $5 \% \mathrm{CO}_{2}$ and $37{ }^{\circ} \mathrm{C}$. The medium consisted of brain heart infusion supplemented with $0.2 \%$ sucrose. After incubation for $8 \mathrm{~h}$, the disks were transferred to new 24 -well plates with fresh medium. ${ }^{21-23}$ After $16 \mathrm{~h}$, the disks were transferred to new 24-well plates and incubated for $24 \mathrm{~h}$. This totaled 2 days which were shown previously to form biofilms on resin specimens. ${ }^{21-23}$ The biofilms on the disks were washed three times with phosphate-buffered saline to remove loose 


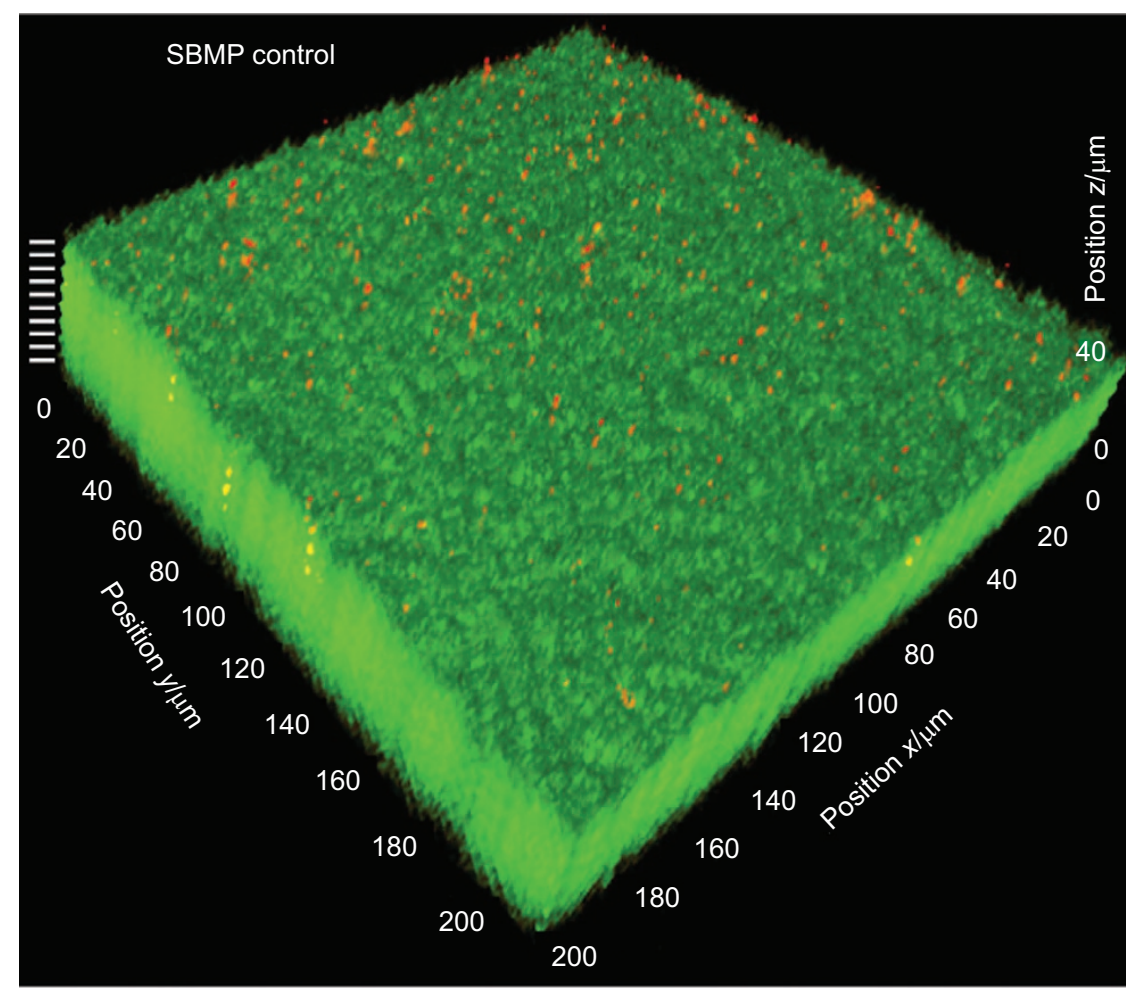

Figure 1 Representative CLSM image of biofilm on SBMP control. The biofilm section parallel to the resin surface is referred to as the $x-y$ plane. The direction perpendicular to resin surface is termed the zaxis. The 2-day biofilm on SBMP control had a thickness of approximately $41 \mu \mathrm{m}$. Ten planes at equal distances along the $z$ axis of the biofilm (indicated by the 10 white lines at the upper left corner) were imaged by CLSM. These 2D images were stacked to reconstruct the 3D biofilm image. 2D, two-dimensional; 3D, three-dimensional; CLSM, confocal laser scanning microscopy; SBMP, Scotchbond multi-purpose bonding agent.

bacteria, and then stained using a BacLight live/dead kit (Molecular Probes, Eugene, OR, USA). Live bacteria were stained with Syto 9 to produce a green fluorescence. Bacteria with compromised membranes were stained with propidium iodide to produce a red fluorescence. ${ }^{21-23}$

The biofilms were investigated using a 3D model as previously described. ${ }^{42}$ The fluorescence was examined visualized using a CLSM (LSM510; Carl Zeiss, Thornwood, NY, USA). Green fluorescence was provided with an argon laser (488-nm laser excitation) and red fluorescence was given with a helium-neon laser (543 nm laser excitation). Images were taken from the bottom of the biofilm that was in contact with the resin disk surface, section by section to the top surface of the biofilm. For the purpose of illustration, an example of a biofilm on the SBMP control disk is shown in Figure 1. The biofilm section parallel to the resin surface was referred to as the $x-y$ plane, and the direction perpendicular to the resin surface is called the $z$ axis. For each biofilm, 10 planes at equal distances (indicated by the 10 white lines at the upper left corner in Figure 1) along the $z$ axis were imaged to obtain an overall view of the biofilm volume. These $2 \mathrm{D}$ sections were stacked and reconstructed into a 3D image of the biofilm using the IMARIS software (Bitplane, Saint Paul, MN, USA). The biofilm images were analyzed using a software (bioImageL; Faculty of Odontology, Malmö University, Malmö, Sweden). ${ }^{38}$ The bioImageL software is based on color segmentation algorithms written in MATLAB (MathWorks, Natick, MA, USA) and is able to produce information of the structure and spatial differences in the biofilm. The biofilm is characterized by parameters including biofilm thickness, green-stained live bacteria volume, red-stained dead bacteria volume, as well as the live and dead bacteria coverage on each twodimensional $x-y$ section in the biofilm.

\section{Statistical analysis}

Statistical analyses were performed using SPSS 17.0 software (SPSS, Chicago, IL, USA). One way analyses of variance were used to detect the significant effects of the variables using a $P$ value of 0.05 .

\section{RESULTS}

A typical CLSM image of a 3D biofilm on SBMP control is shown in Figure 1 as described in the section on 'CLSM analysis of biofilms'. Figure 2 shows representative images of biofilms on the six bonding agents containing QAM with various CL. Live bacteria were stained green, and dead bacteria were stained red. SBMP control and that with CL3 had primarily live bacteria. The amount of dead bacteria gradually increased when CL was increased to 6, 9 and 12. When CL was increased to 16 , the biofilms were primarily dead with red staining. When CL was further increased to 18 , there was an increase in green staining of live bacteria. Furthermore, the 3D images highlighted the differences in biofilm thickness, with biofilms on SBMP control being the thickest and that of CL16 being the thinnest.

The biofilm thickness results are quantified in Figure 3 (mean \pm s.d.; $n=6$ ). Biofilm thickness steadily decreased from SBMP control to those containing QAM with increasing $\mathrm{CL}$, reaching a minimum at CL16. When CL was further increased to 18 , the biofilm thickness increased. Values indicated by dissimilar letters are significantly different from each other $(P<0.05)$. 

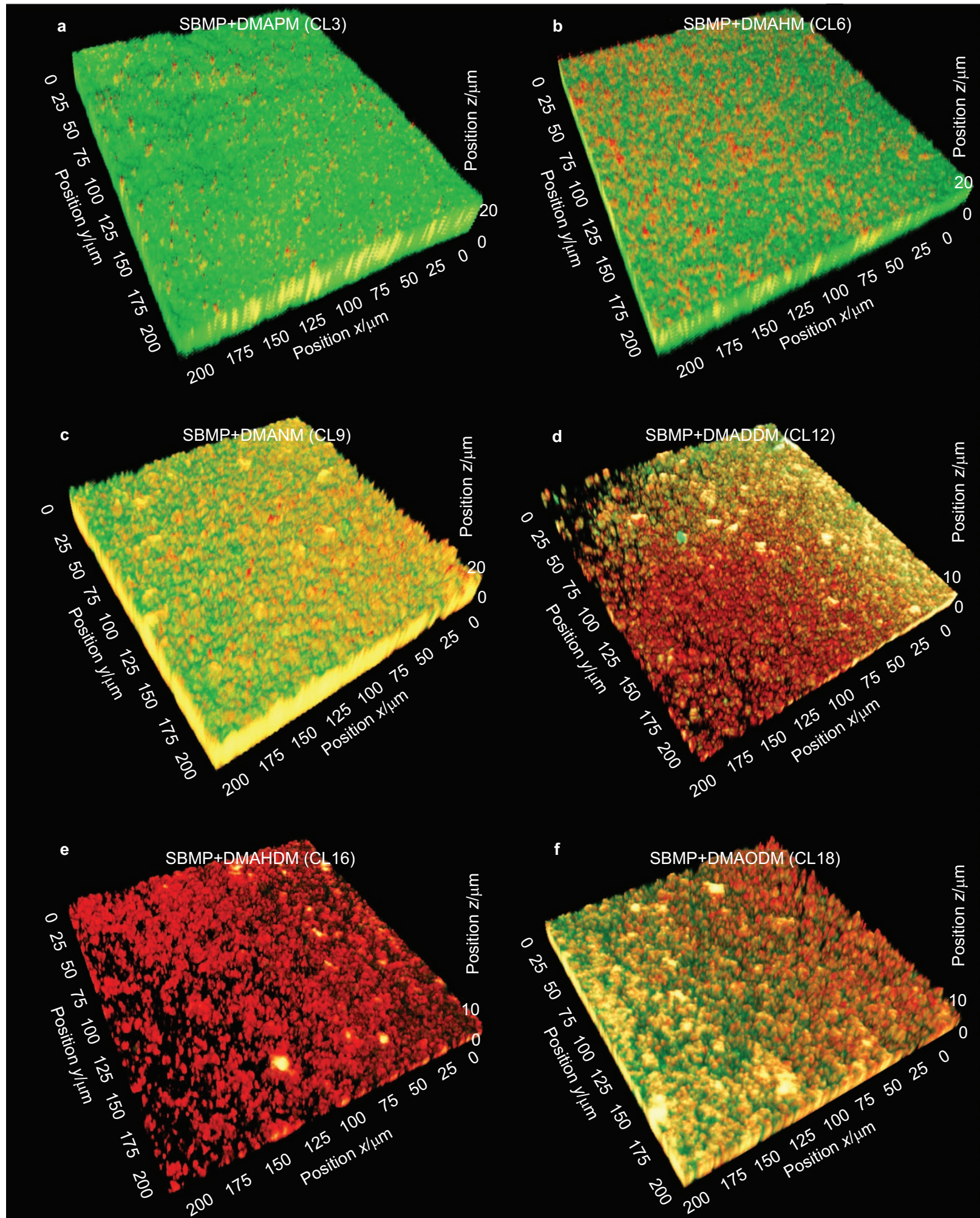

Figure 2 Typical 3D images of biofilms cultured for 2 days on bonding agents. (a) SBMP+DMAPM (CL3); (b) SBMP+DMAHM (CL6); (c) SBMP+DMANM (CL9); (d) SBMP+DMADDM (CL12); (e) SBMP+DMAHDM (CL16); (f) SBMP+DMAODM (CL18). Live bacteria were stained green and dead bacteria were stained red. CL, chain length; 3D, three-dimensional; DMADDM, dimethylaminododecyl methacrylate; DMAHDM, dimethylaminohexadecyl methacrylate; DMAHM, dimethylaminohexyl methacrylate; DMANM, dimethylaminononyl methacrylate; DMAODM, dimethylaminooctadecyl methacrylate; DMAPM, dimethylaminopropyl methacrylate; SBMP, Scotchbond multi-purpose bonding agent. 


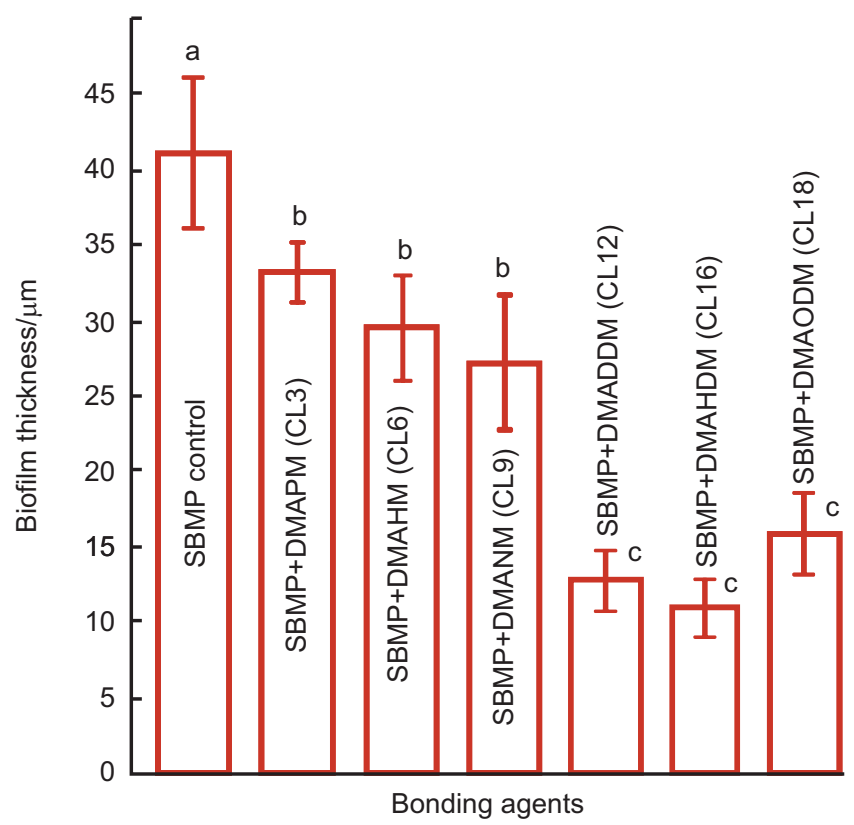

Figure 3 Biofilm thickness cultured for 2 days on bonding agents (mean \pm s.d.; $\boldsymbol{n}=6$ ). Seven bonding agents were tested: SBMP control, SBMP+DMAPM (CL3), SBMP+DMAHM (CL6), SBMP+DMANM (CL9), SBMP+DMADDM (CL12), SBMP+DMAHDM (CL16) and SBMP+DMAODM (CL18). Values indicated by dissimilar letters are significantly different from each other $(P<0.05)$. CL, chain length; 3D, three-dimensional; DMADDM, dimethylaminododecyl methacrylate; DMAHDM, dimethylaminohexadecyl methacrylate; DMAHM, dimethylaminohexyl methacrylate; DMANM, dimethylaminononyl methacrylate; DMAODM, dimethylaminooctadecyl methacrylate; DMAPM, dimethylaminopropyl methacrylate; SBMP, Scotchbond multi-purpose bonding agent; s.d., standard deviation.

The biofilm volume results are plotted in Figure 4: live biofilm volume (Figure $4 \mathrm{a}$ ) and dead biofilm volume (mean \pm s.d.; $n=6$ ) (Figure $4 \mathrm{~b}$ ). SBMP control had the greatest live biofilm volume. The live biofilm volume gradually decreased with increasing CL, reaching a minimum at CL16, and then increased at CL18. The dead biofilm volume first increased with increasing $\mathrm{CL}$ and then reached a plateau. CL16 had the least live biofilm volume which was two orders of magnitude lower than that of SBMP control.
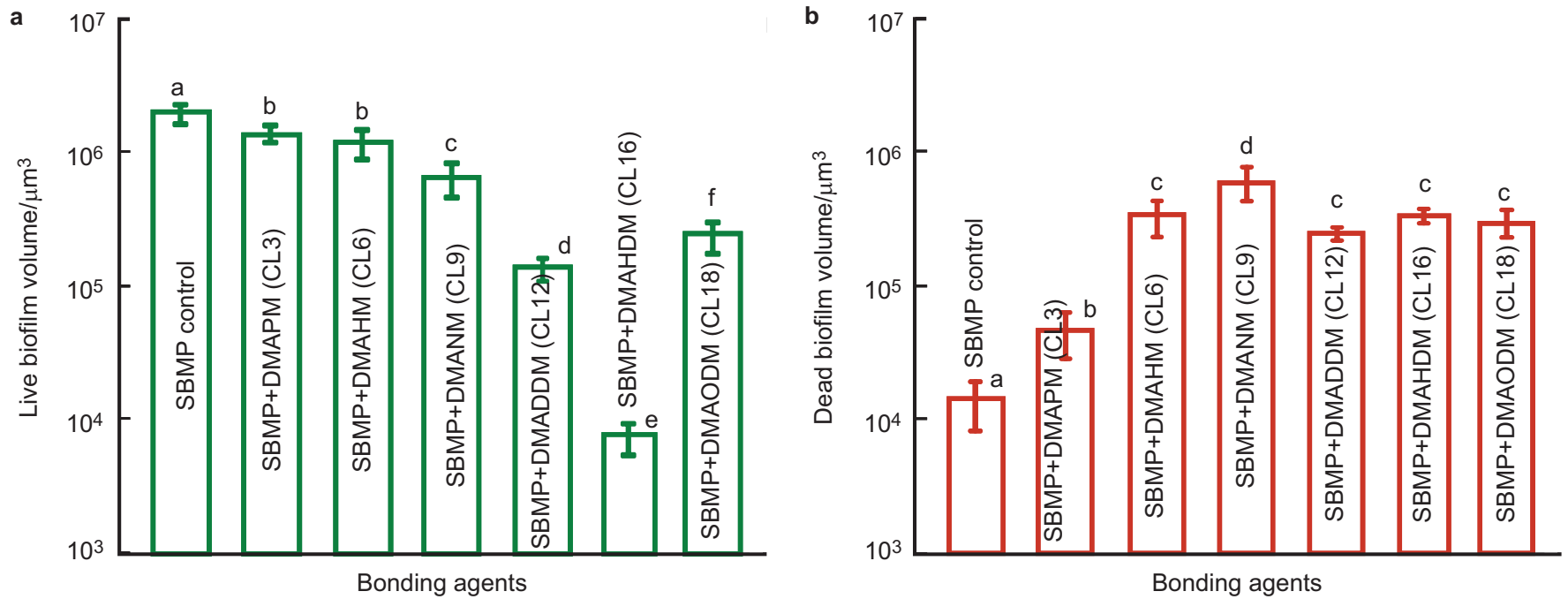

Figure 4 Biofilm volume cultured for 2 days on bonding agents. (a) Live biofilm volume; (b) dead biofilm volume (mean \pm s.d.; $n=6$ ). Seven bonding agents were tested: SBMP control, SBMP+DMAPM (CL3), SBMP+DMAHM (CL6), SBMP+DMANM (CL9), SBMP+DMADDM (CL12), SBMP+DMAHDM (CL16) and SBMP+DMAODM (CL18). In each plot, values with dissimilar letters are significantly different from each other $(P<0.05)$. CL, chain length; 3D, three-dimensional; DMADDM, dimethylaminododecyl methacrylate; DMAHDM, dimethylaminohexadecyl methacrylate; DMAHM, dimethylaminohexyl methacrylate; DMANM, dimethylaminononyl methacrylate; DMAODM, dimethylaminooctadecyl methacrylate; DMAPM, dimethylaminopropyl methacrylate; SBMP, Scotchbond multi-purpose bonding agent; s.d., standard deviation. 


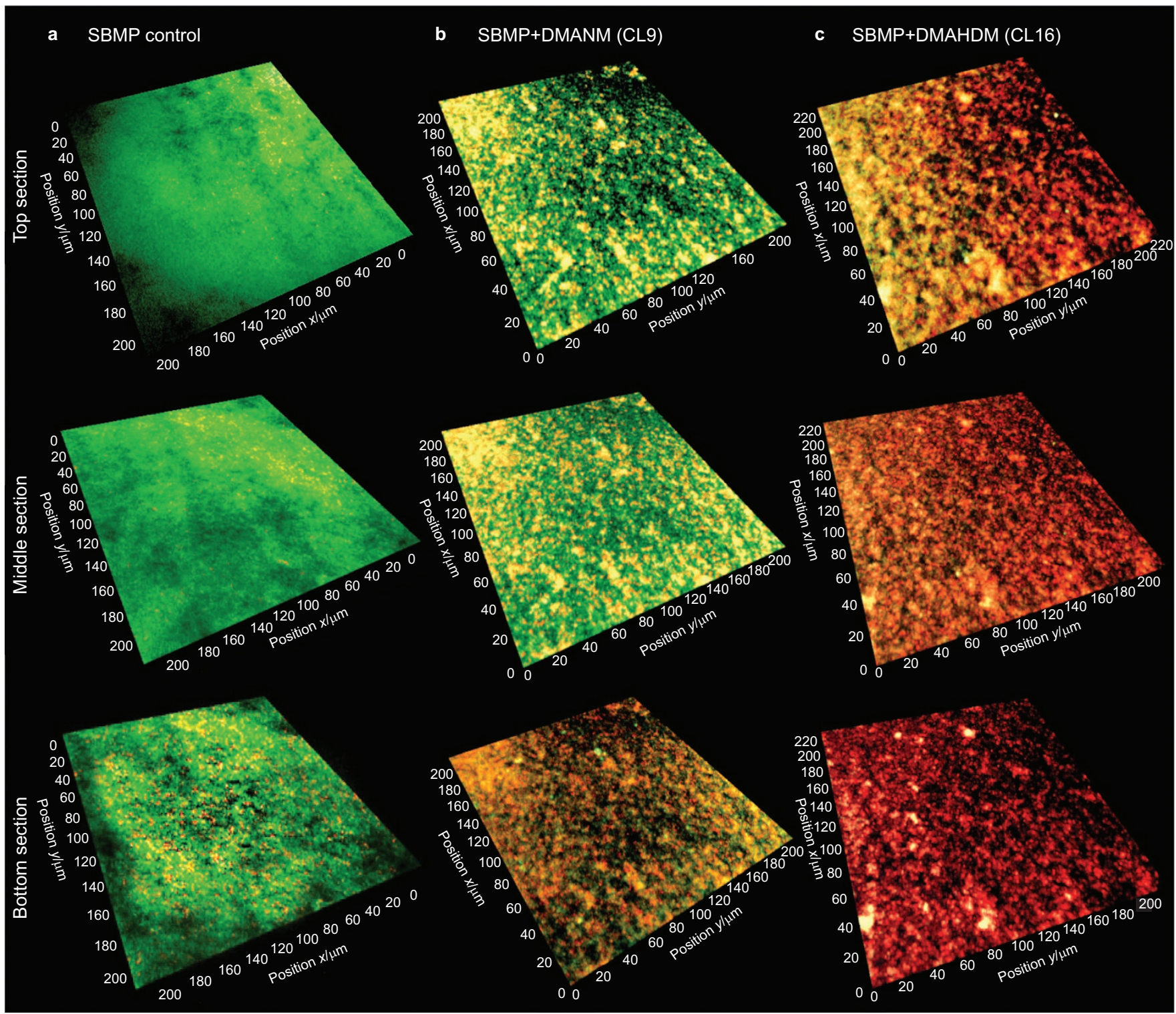

Figure 5 Representative 2D live/dead staining images of cross-sectioned biofilm in the $\boldsymbol{x}-\boldsymbol{y}$ plane. All seven bonding agents were tested; shown here are three examples: control, QAM with an intermediate CL of the most potent QAM. The top labels indicate the materials: SBMP control, SBMP+DMANM (CL9) and SBMP+DMAHDM (CL16). The left labels indicate the location of the section in the biofilm: the top surface, the middle section and the bottom section (near the resin surface) of the biofilm. Live bacteria were stained green and dead bacteria were stained red. CL, chain length; 2D, two-dimensional; DMAHDM, dimethylaminohexadecyl methacrylate; DMAHM, dimethylaminohexyl methacrylate; DMANM, dimethylaminononyl methacrylate; QAM, quaternary ammonium methacrylate; SBMP, Scotchbond multi-purpose bonding agent.

Typical live/dead staining images of $2 \mathrm{D} x-y$ sections are shown in Figure 5 for the top surface, the middle section and the bottom section (near the resin surface) of the biofilm. Three materials are shown in Figure 5 as examples: SBMP control (Figure 5a), SBMP+DMANM (CL9) (Figure 5b) and SBMP+DMAHDM (CL16) (Figure 5c). The top surface of SBMP control biofilm had the most live bacteria, while its bottom surface had a slight increase in dead bacteria amount. For SBMP+ DMANM (CL9), the bottom of biofilm had noticeably more dead bacteria and the top section of the biofilm had more live bacteria. For SBMP+ DMAHDM (CL16), there appeared to be predominantly compromised bacteria throughout the biofilm thickness, although there appeared to be a small amount of live bacteria in the top section of the biofilm.
The biofilm vitality distribution in the different layers of the biofilm $v s$. biofilm height is shown in Figure 6 (mean \pm s.d.; $n=6$ ). The vertical axis shows the percentage of live bacteria measured from $2 \mathrm{D}$ sections such as those in Figure 5. The horizontal axis indicates the biofilm thickness at which the 2D image was taken. SBMP control group and the group with CL3 had similar results; hence, the CL3 group was not included in Figure 6 to save space. SBMP control had a percentage of live bacteria of $63 \%$ at the biofilm bottom; it gradually increased and approached $100 \%$ near the top of the biofilm. At CL of 6 and 9, the percentage of live bacteria at the biofilm bottom decreased to $60 \%$ and $37 \%$, respectively. However, the percentage of live bacteria was still nearly $100 \%$ at the top of the biofilm. At CL of 12 , not only did the 


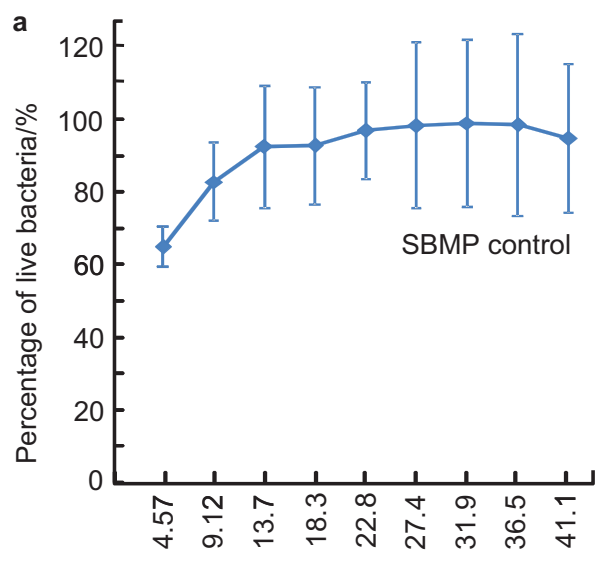

Biofilm thickness from resin surface/ $\mu \mathrm{m}$

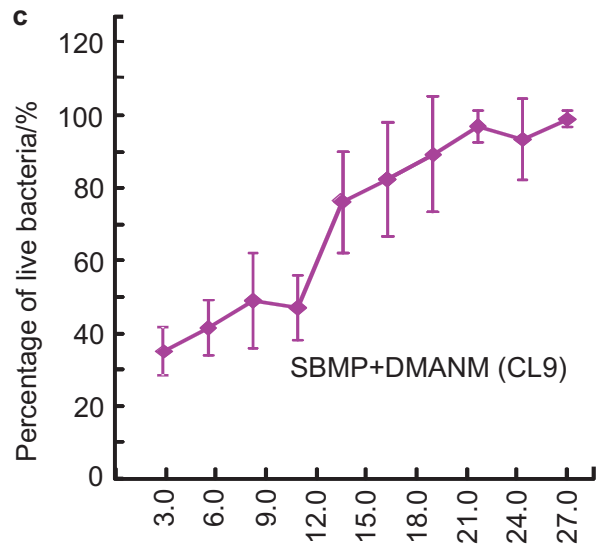

Biofilm thickness from resin surface/ $\mu \mathrm{m}$

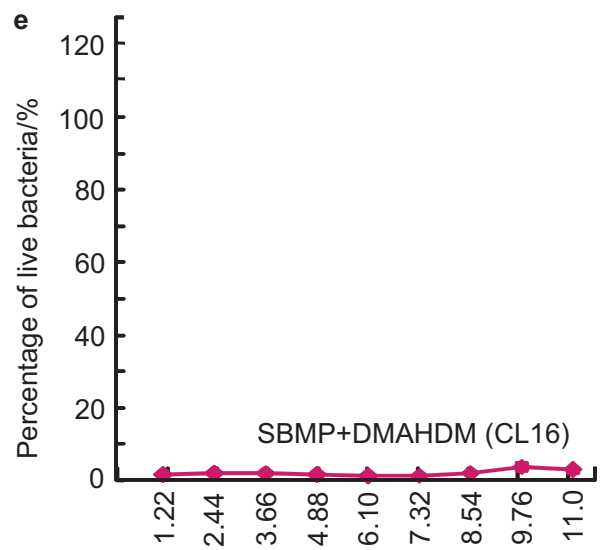

Biofilm thickness from resin surface/ $\mu \mathrm{m}$

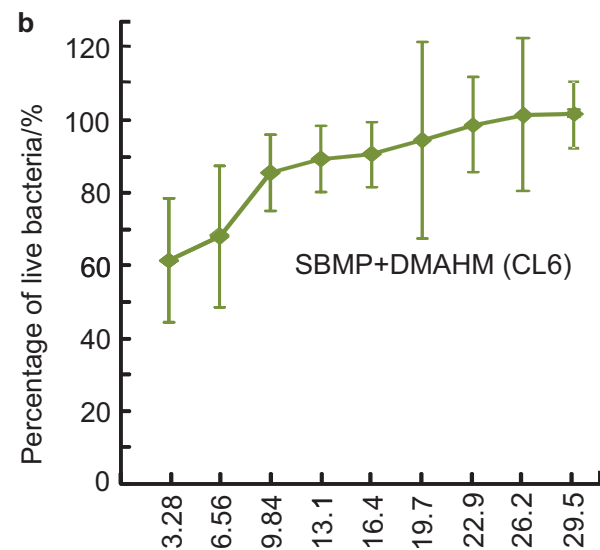

Biofilm thickness from resin surface/ $\mu \mathrm{m}$

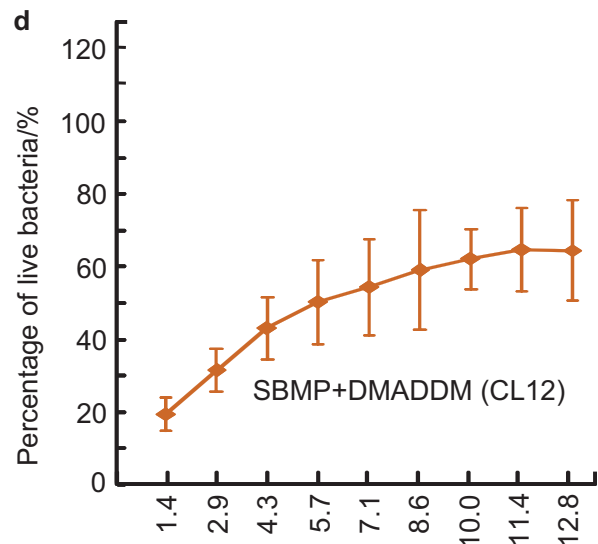

Biofilm thickness from resin surface $/ \mu \mathrm{m}$

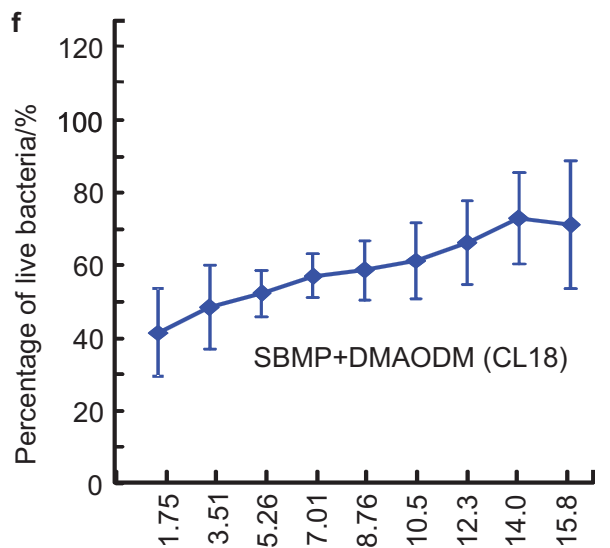

Biofilm thickness from resin surface/ $\mu \mathrm{m}$

Figure 6 Viability distribution in biofilm (mean \pm s.d.; $\boldsymbol{n = 6}$ ). The percentage of live bacteria was measured from 2D images (Figure 5). Percentage of live bacteria =live bacteria area/(live bacteria area+dead bacteria area). Percentage of live bacteria is plotted vs. location of 2D image in biofilm at distance from resin surface. (a) SBMP control; (b) SBMP+DMAHM (CL6); (c) SBMP+DMANM (CL9); (d) SBMP+DMADDM (CL12); (e) SBMP+DMAHDM (CL16); (f) SBMP+DMAODM (CL18). The curve for SBMP+DMAPM (CL3) is similar to SBMP control and is not included. CL, chain length; 2D, two-dimensional; DMADDM, dimethylaminododecyl methacrylate; DMAHDM, dimethylaminohexadecyl methacrylate; DMAHM, dimethylaminohexyl methacrylate; DMANM, dimethylaminononyl methacrylate; DMAODM, dimethylaminooctadecyl methacrylate; DMAPM, dimethylaminopropyl methacrylate; SBMP, Scotchbond multi-purpose bonding agent; s.d., standard deviation.

percentage of live bacteria decrease to $19 \%$ at the biofilm bottom, but the top only had $63 \%$. At CL16, the percentage of live bacteria was close to $0 \%$ throughout the biofilm thickness. When CL was further increased to 18 , biofilm percentage of live bacteria increased.
These results show that: (i) most of the tested antibacterial bonding agents could only inhibit bacteria close to the resin surface and the antibacterial efficacy decreased in biofilm away from resin surface; and (ii) the bonding agent containing DMAHDM 
with CL16 maintained a low viability of nearly $0 \%$ throughout the biofilm.

\section{DISCUSSION}

The present study investigated the 3D biofilm live/dead volume and viability variation in biofilm thickness on dental bonding agents as a function of alkyl chain length for the first time. S. mutans biofilms were examined using a 3D digital reconstruction technique combined with quantitative image analysis. QAMs with CL varying from 3 to 18 were incorporated into bonding agent which exerted a significant anti-biofilm activity. Increasing the CL of bonding agent achieved a stronger effect in reducing the biofilm viability, evidenced by changes in biofilm structure with decreases in biofilm thickness, live biofilm volume and percentage of live bacteria. Oral bacteria in vivo colonize on the toothrestoration surfaces to form biofilms and cariogenic bacteria such as $S$. mutans in the biofilm can metabolize carbohydrates to produce organic acids. This plays an important role in the development of tooth decay and secondary caries at the tooth-restoration margins. Furthermore, within the biofilm, S. mutans display properties that are dramatically distinct from their planktonic counterparts, including much higher resistance to antibacterial agents, which makes the biofilm much more difficult to kill than planktonic bacteria. Therefore, the antibacterial bonding agent containing the new QAMs, especially that using CL16 with effective killing of the biofilm, could be beneficial in caries-inhibiting dental applications.

This study showed a strong CL dependence of anti-biofilm properties of bonding agent. The mechanism of QAS to kill bacteria is believed to involve the alteration of membrane permeability or surface electrostatic balance of bacteria, thus causing cytoplasmic leakage. ${ }^{17,26-29}$ It has also been noticed that the alkyl chain length has a significant effect on biocidal activity since long cationic polymers may interact more effectively with the cytoplasmic membranes. ${ }^{28-29}$ The present study revealed that the 3D structural changes in biofilms were associated with the CL of QAM in bonding agent. With CL increasing from 3 to 16 , the biofilm thickness, live bacteria volume and the percentage of live bacteria all decreased. However, after reaching the maximum antimicrobial ability with CL16, no further strengthening of bacteria-inhibition effect was detected with increasing the CL to 18. A similar phenomenon was observed in previous studies which were not on dental bonding agents. ${ }^{18,43-44}$ This was explained as a cutoff effect. ${ }^{45}$ Among the various assumptions proposed to explain the origin of the cutoff effect, the concept of free volume could be applied to QAS. ${ }^{44}$ Free volume is the unoccupied space between molecules. In solution or culture medium, the polar ammonium heads will interact with those of phospholipids of the bacteria and their hydrocarbon chains will orient parallel to the hydrocarbon chains of phospholipids. ${ }^{44}$ The hydrocarbon chains are parallel to those of phopholipids of the cell. In this case, the density of the bilayer hydrophobic region is necessarily altered and a free volume is formed. When the hydrocarbon chain of the QAS is shorter than that of phospholipids, the total free volume generated in the bilayer is small. When the hydrocarbon chain length of QAS becomes comparable to that of phospholipids, the free volume drops off and tends toward zero. Molecules containing chains between these two extremes lead to the most essential free volume inside the bilayer. ${ }^{44}$ The larger the free volume, the more the membrane of bacteria is expected to be disrupted and the bactericidal activity is enhanced. Hence, the present study indicates that CL16 with the maximum antibacterial activity may possess the largest free volume in the bilayer.
Furthermore, CLSM examination of the present study showed that for SBMP control, the bottom layer of the biofilm adjacent to resin contained a higher proportion of nonviable bacteria than the upper layer of biofilm in contact with culture medium. This was likely because the deeper layer of the biofilm had less access to oxygen, a lower availability of primary nutrients and more secondary metabolites accumulation than the outer layer of the biofilm. For bonding agents containing QAMs, the compromised bacteria were more concentrated in the lower layer of the biofilm, and the viability percentage increased with the biofilm height away from the resin surface. This was likely related to the contact-killing mechanism of QAM resin in which the QAM was copolymerized with and immobilized in the resin. Hence, due to contact-killing effect, the bactericidal efficacy is decreased away from the surface due to a lack of contact. For example, for CL 9 in Figure 6, the percentage of live bacteria was only 37\% near the antibacterial resin surface, but close to $100 \%$ away from the resin surface near the biofilm top.

For the bonding agent containing DMAHDM with CL16, the biofilm consisted mainly of dead microorganisms throughout the biofilm thickness. This may suggest another possible antibacterial mechanism. Previous studies suggested that a stress condition or challenge in bacteria could trigger a built-in suicide program in the biofilm, ${ }^{46-47}$ which was also called programmed cell death. ${ }^{46}$ Being challenged by bactericidal agents may serve as a trigger for programmed cell death in the surrounding bacteria. ${ }^{46-47}$ Indeed, the present study showed that the bonding agent at CL16 killed the entire biofilm. This is consistent with a previous in vivo study showing that the biofilm on a QAM composite intraorally in human participants was dead not only on the resin surface, but also in the outer, more remote parts of the biofilm. ${ }^{48}$ This was explained as due to an intracellularly mediated death program, in which the bacterial lysis by QAM on the resin surface may function as a stressful condition triggering programmed cell death to the bacteria further away in the biofilm. ${ }^{48}$ Further study is needed to investigate if there is significant leachout of QAM from the resin which might contribute to killing bacteria at a distance away from the resin surface. In the present study, the resin disks were agitated in $200 \mathrm{~mL}$ of water via magnetic stirring for $1 \mathrm{~h}$ to remove possible uncured monomers, following a previous study. ${ }^{15}$ Further study should water-age the disks for long periods of time such as 6 months, ${ }^{49}$ and then inoculate bacteria to determine if the antibacterial activity is durable and if the bonding agent with CL16 can still kill the entire 3D biofilm. Nonetheless, the results of the present study such as Figure 6 clearly show the effect of CL in bonding agent on 3D biofilm properties, as all the disks were prepared and treated in the same manner.

Regarding potential applications of the bonding agent containing DMAHDM with CL16, recurrent caries at the tooth-restoration margins is a primary reason for restoration failure. Hence, the antibacterial bonding agent in the uncured state could flow into dentinal tubules and kill residual bacteria in the tooth cavity. ${ }^{14-15,24,50}$ The antibacterial bonding agent in the cured state could inhibit bacteria invasion along the margins, which could be especially useful to inhibit bacteria when marginal microgaps occur during service. ${ }^{14-15,24,50}$ Furthermore, there has been an increased interest in the less removal of tooth structure and minimal intervention dentistry. ${ }^{51}$ While the treatment could preserve tooth structure, it could also leave behind more carious tissues with active bacteria. Atraumatic restorative treatment does not remove the carious tissues completely, leaving remnants of lesions and bacteria. ${ }^{52}$ Therefore, these applications could potentially benefit from the bonding agent containing DMAHDM. In addition, DMAHDM could also be promising for incorporation into composites for 
antibacterial restorations. ${ }^{53-54}$ The materials need to be biocompatible for clinical applications. A recent study showed that the fibroblast viability and odontoblast viability of DMAHDM were better than bisphenolglycerolate dimethacrylate. ${ }^{30}$ The eluents from the cured resin containing DMAHDM caused fibroblast viability and odontoblast viability that were not significantly different from the resin control without DMAHDM. ${ }^{30}$ Further studies are needed to investigate the potential dental applications DMAHDM-containing resins and composites.

\section{CONCLUSIONS}

This study investigated the effect of CL on 3D biofilm structure and live/dead viability variation $v s$. biofilm thickness on dental bonding agent for the first time. The results showed that: (i) strong antibacterial function was achieved by adding QAM into bonding agent; (ii) biofilm thickness and live volume decreased with increasing CL from 3 to 16 , but then increased at CL of 18; (iii) except for CL16, antibacterial bonding agents with all tested CL values could only inhibit bacteria close to resin surface and the antibacterial efficacy decreased in the biofilm away from resin surface; (iv) the bonding agent containing DMAHDM with CL 16 yielded a percentage of live bacteria of close to $0 \%$ throughout the biofilm thickness. The $3 \mathrm{D}$ biofilm analysis via CLSM and digital reconstruction method is useful for understanding biofilm-resin interactions and antibacterial resin effects on biofilm structure and $3 \mathrm{D}$ viability distribution. Based on the specific resin formulations tested in this study, antibacterial bonding agents with CL16 are useful for a wide range of dental applications to combat bacteria and biofilms at tooth-restoration margins to inhibit secondary caries.

\section{DISCLAIMER}

Certain commercial materials and equipment are identified to specify experimental procedures. This does not imply recommendation by National Institute of Standards and Technology (NIST)/American Dental Association (ADA).

\section{ACKNOWLEDGEMENTS}

We thank Dr Lei Cheng, Dr Fang Li, Dr Nancy J Lin and Dr Sheng Lin-Gibson for discussions. We are grateful to Dr Dean Dessem for help with the confocal laser scanning microscope. This study was supported by NIH R01 DE17974 (Hockin H K Xu), West China School of Stomatology (Han Zhou) and a Seed Grant from Department of Endodontics, Prosthodontics and Operative Dentistry, University of Maryland (Hockin H K Xu).

1 Drummond JL. Degradation, fatigue, and failure of resin dental composite materials. $J$ Dent Res 2008; 87(8): 710-719.

2 Lynch CD, Frazier KB, McConnell RJ et al. State-of-the-art techniques in Operative Dentistry: contemporary teaching of posterior composites in UK and Irish dental schools. Br Dent J 2010; 209(3): 129-136.

3 Ferracane JL. Resin composite — state of the art. Dent Mater 2011; 27(1): 29-38.

4 Demarco FF, Correa MB, Cenci MS et al. Longevity of posterior composite restorations: not only a matter of materials. Dent Mater 2012; 28(1): 87-101.

5 Spencer $P, Y e$ Q, Park JG et al. Adhesive/dentin interface: the weak link in the composite restoration. Ann Biomed Eng 2010; 38(6): 1989-2003.

6 Ferracane JL, Hilton TJ, Sakaguchi RL. Introduction to and outcomes of the conference on adhesion in dentistry. Dent Mater 2010; 26(2): 105-107.

7 Pashley DH, Tay FR, Breschi L et al. State of the art etch-and-rinse adhesives. Dent Mater 2011; 27(1): 1-16.

8 Mjor IA, Toffeneti F. Secondary caries: a literature review with caries reports. Quintessence Int 2000; 31(3): 165-179.

9 Sakaguchi RL. Review of the current status and challenges for dental posterior restorative composites: clinical, chemistry, and physical behavior considerations. Dent Mater 2005; 21(1): 3-6.

10 Frost PM. An audit on the placement and replacement of restorations in a general dental practice. Prim Dent Care 2002; 9(1): 31-36.

11 Bagramian RA, Garcia-Godoy F, Volpe AR. The global increase in dental caries. A pending public health crisis. Am J Dent 2009; 22(1): 3-8.
12 ten Cate JM. Biofilms, a new approach to the microbiology of dental plaque. Odontology 2006; 94(1): 1-9.

13 Imazato S. Review: antibacterial properties of resin composites and dentin bonding systems. Dent Mater 2003; 19(6): 449-457

14 Imazato S. Bioactive restorative materials with antibacterial effects: new dimension of innovation in restorative dentistry. Dent Mater J 2009; 28(1): 11-19.

15 Imazato S, Ehara A, Torii M et al. Antibacterial activity of dentine primer containing MDPB after curing. J Dent 1998; 26(3): 267-271.

$16 \mathrm{Li} \mathrm{F}$, Chen J, Chai Z et al. Effects of a dental adhesive incorporating antibacterial monomer on the growth, adherence and membrane integrity of Streptococcus mutans. J Dent 2009; 37(4): 289-296.

17 Namba N, Yoshida Y, Nagaoka N et al. Antibacterial effect of bactericide immobilized in resin matrix. Dent Mater 2009; 25(4): 424-430.

18 Xie D, Weng Y, Guo X et al. Preparation and evaluation of a novel glass-ionomer cement with antibacterial functions. Dent Mater 2011; 27(5): 487-496.

$19 \mathrm{Xu}$ X, Wang Y, Liao S et al. Synthesis and characterization of antibacterial dental monomers and composites. J Biomed Mater Res B Appl Biomater 2012; 100(4): 1151-1162.

20 Antonucci JM, Zeiger DN, Tang $K$ et al. Synthesis and characterization of dimethacrylates containing quaternary ammonium functionalities for dental applications. Dent Mater 2012; 28(2): 219-228.

21 Cheng L, Weir MD, Xu HH et al. Antibacterial amorphous calcium phosphate nanocomposite with quaternary ammonium salt and silver nanoparticles. Dent Mater 2012; 28(5): 561-572.

22 Cheng L, Zhang K, Melo MA et al. Anti-biofilm dentin primer with quaternary ammonium and silver nanoparticles. J Dent Res 2012; 91(6): 598-604.

23 Zhang $\mathrm{K}$, Melo MA, Cheng $\mathrm{L}$ et al. Effect of quaternary ammonium and silver nanoparticle-containing adhesives on dentin bond strength and dental plaque microcosm biofilms. Dent Mater 2012; 28(8): 842-852.

24 Hiraishi N, Yiu CK, King NM et al. Effect of chlorhexidine incorporation into a selfetching primer on dentine bond strength of a luting cement. J Dent 2010; 38(6): 496502.

25 Tezvergil-Mutluay A, Agee KA, Uchiyama T et al. The inhibitory effects of quaternary ammonium methacrylates on soluble and matrix-bound MMPs. J Dent Res 2011; 90(4): 535-540

26 Beyth N, Yudovin-Farber I, Bahir R et al. Antibacterial activity of dental composites containing quaternary ammonium polyethylenimine nanoparticles against Streptococcus mutans. Biomaterials 2006; 27(21): 3995-4002.

27 Lin J, Qiu S, Lewis K et al. Bactericidal properties of flat surfaces and nanoparticles derivatized with alkylated polyethylenimines. Biotechnol Prog 2002; 18(5): 1082 1086.

28 Tiller JC, Liao CJ, Lewis $\mathrm{K}$ et al. Designing surfaces that kill bacteria on contact. Proc Natl Acad Sci U S A 2001; 98(11): 5981-5985.

29 Murata H, Koepsel RR, Matyjaszewski K et al. Permanent, non-leaching antibacteria surfaces-2: how high density cationic surfaces kill bacterial cells. Biomaterials 2007; 28(32): 4870-4879.

30 Li F, Weir MD, Xu HK. Effects of quaternary ammonium chain length on antibacterial bonding agents. J Dent Res 2013; 92(10): 932-938.

31 Brecx M, Winkler M, Netuschil L. Human dental plaque formation on plastic films. A quantitative SEM study. J West Soc Periodontol Periodontal Abstr 1994; 42(3): 77-80.

32 Hannig M. Transmission electron microscopy of early plaque formation on dental materials in vivo. Eur J Oral Sci 1999; 107(1): 55-64.

33 Wood S, Kirkham J, Marsh P et al. Architecture of intact natural human plaque biofilms studied by confocal laser scanning microscopy. J Dent Res 2000; 79(1): 21-27.

34 Robinson C, Kirkham J, Percival R et al. A method for the quantitative site-specific study of the biochemistry within dental plaque biofilms formed in vivo. Caries Res 1997; 31(3): 194-200.

35 Singleton S, Treloar R, Warren P et al. Methods for microscopic characterization of oral biofilms: analysis of colonization, microstructure, and molecular transport phenomena. Adv Dent Res 1997; 11(1): 133-149.

36 Gong SQ, Epasinghe J, Rueggeberg FA et al. An ORMOSIL-containing orthodontic acrylic resin with concomitant improvements in antimicrobial and fracture toughness properties. PLoS One 2012; 7(8): e42355.

37 Gong SQ, Epasinghe DJ, Bin Zhou B et al. Effect of water-aging on the antimicrobial activities of an ORMOSIL-containing orthodontic acrylic resin. Acta Biomater 2013; 9(6): 6964-6973.

38 Chávez de Paz LE. Image analysis software based on color segmentation for characterization of viability and physiological activity of biofilms. App/ Environ Microbiol 2009; 75(6): 1734-1739.

39 Auschill T, Artweiler N, Netuschil L et al. Spatial distribution of vital and dead microorganisms in dental biofilms. Arch Oral Biol 2001; 46(5): 471-476.

40 Netuschil L, Reich E, Unteregger G et al. A pilot study of confocal laser scanning microscopy for the assessment of undisturbed dental plaque vitality and topography. Arch Oral Biol 1998; 43(4): 277-285.

41 Cheng L, Weir MD, Zhang $\mathrm{K}$ et al. Dental primer and adhesive containing a new antibacterial quaternary ammonium monomer dimethylaminododecyl methacrylate. J Dent 2013; 41(4): 345-355.

42 Chávez de Paz LE, Resin A, Howard KA et al. Antimicrobial effect of chitosan nanoparticles on streptococcus mutans biofilms. Appl Environ Microbiol 2011; 77(11): 3892-3895.

43 Thebault P, Taffin de Givenchy E, Levy R et al. Preparation and antimicrobial behaviour of quaternary ammonium thiol derivatives able to be grafted on metal surfaces. Eur J Med Chem 2009; 44(2): 717-724. 
$44 \mathrm{He} J$, Söderling E, Österblad M et al. Synthesis of methacrylate monomers with antibacterial effects against S. mutans. Molecules 2011; 16(11): 97559763.

45 Balgavý P, Devínsky F. Cut-off effects in biological activities of surfactants. Adv Colloid Interface Sci 1996; 66: 23-63.

46 Engelberg-Kulka $\mathrm{H}$, Amitai S, Kolodkin-Gal I et al. Bacterial programmed cell death and multicellular behavior in bacteria. PLoS Genet 2006; 2(10): e135.

47 Gerdes K, Christensen SK, Løbner-Olesen A. Prokaryotic toxin-antitoxin stress response loci. Nat Rev Microbiol 2005; 3(5): 371-382.

48 Beyth N, Yudovin-Farber I, Bahir R et al. Polyethyleneimine nanoparticles incorporated into resin composite cause cell death and trigger biofilm stress in vivo. Proc Natl Acad Sci U S A 2010; 107(51): 22038-22043.

49 Zhang K, Cheng L, Wu EJ et al. Effect of water-aging on dentin bond strength and antibiofilm activity of bonding agent containing antibacterial monomer dimethylaminododecyl methacrylate. J Dent 2013; 41(6): 504-513.

50 Imazato S, Kinomoto $\mathrm{Y}$, Tarumi $\mathrm{H}$ et al. Antibacterial activity and bonding characteristics of an adhesive resin containing antibacterial monomer MDPB. Dent Mater 2003; 19(4): 313-319.
51 Lynch CD, Frazier KB, McConnell RJ et al. Minimally invasive management of denta caries: contemporary teaching of posterior resin-based composite placement in U.S. and Canadian dental schools. J Am Dent Assoc 2011; 142(6): 612-620.

52 Frencken JE, van't Hof MA, van Amerongen WE et al. Effectiveness of single-surface ART restorations in the permanent dentition: a meta-analysis. J Dent Res 2004; 83(2): 120-123.

53 Imazato S, Torii M, Tsuchitani Y et al. Incorporation of bacterial inhibitor into resin composite. J Dent Res 1994; 73(8): 1437-1443.

54 Cheng L, Weir MD, Zhang $\mathrm{K}$ et al. Antibacterial nanocomposite with calcium phosphate and quaternary ammonium. J Dent Res 2012; 91(5): 460-466.

(i) $\Theta$ This work is licensed under a Creative Commons Attribution-

BY NC ND NonCommercial-NoDerivs 3.0 Unported License. The images or other third party material in this article are included in the article's Creative Commons license, unless indicated otherwise in the credit line; if the material is not included under the Creative Commons license, users will need to obtain permission from the license holder to reproduce the material. To view a copy of this license, visit http://creativecommons.org/licenses/ by-nc-nd/3.0/ 